\title{
SOME THEOREMS ON THE EXPLICIT EVALUATION OF RAMANUJAN'S THETA-FUNCTIONS
}

\author{
NAYANDEEP DEKA BARUAH and P. BHATTACHARYYA
}

Received 8 October 2001

\begin{abstract}
Bruce C. Berndt et al. and Soon-Yi Kang have proved many of Ramanujan's formulas for the explicit evaluation of the Rogers-Ramanujan continued fraction and theta-functions in terms of Weber-Ramanujan class invariants. In this note, we give alternative proofs of some of these identities of theta-functions recorded by Ramanujan in his notebooks and deduce some formulas for the explicit evaluation of his theta-functions in terms of WeberRamanujan class invariants.
\end{abstract}

2000 Mathematics Subject Classification: 11A55, 11F20, 11 F27.

1. Introduction. Ramanujan's general theta-function $f(a, b)$ is given by

$$
f(a, b)=\sum_{k=-\infty}^{\infty} a^{k(k+1) / 2} b^{k(k-1) / 2},
$$

where $|a b|<1$. If we set $a=q^{2 i z}, b=q^{-2 i z}$, and $q=e^{\pi i \tau}$, where $z$ is complex and $\operatorname{Im}(\tau)>0$, then $f(a, b)=\vartheta_{3}(z, \tau)$, where $\vartheta_{3}(z, \tau)$ denotes one of the classical thetafunctions in its standard notation [9, page 464]. After Ramanujan, we define the following special types of his theta-function.

If $|q|<1$, then

$$
\begin{aligned}
\phi(q) & :=f(q, q)=1+2 \sum_{k=1}^{\infty} q^{k^{2}}, \\
\psi(q) & :=f\left(q, q^{3}\right)=\sum_{k=0}^{\infty} q^{k(k+1) / 2}, \\
f(-q) & :=f\left(-q,-q^{2}\right)=\sum_{k=0}^{\infty}(-1)^{k} q^{k(3 k-1) / 2}+\sum_{k=1}^{\infty}(-1)^{k} q^{k(3 k+1) / 2}, \\
\chi(q) & :=\left(-q ; q^{2}\right)_{\infty},
\end{aligned}
$$

where $(a ; q)_{\infty}:=\prod_{k=0}^{\infty}\left(1-a q^{k}\right)$. The function $\chi(q)$ is only for notational purposes. Also, note that $f(-q)=q^{-1 / 24} \eta(z)$, where $q=e^{2 \pi i z}$ and $\eta$ denotes the Dedekind eta-function. Much of Ramanujan's discoveries about theta-functions can be found in Chapters 1621 of the organized pages of his second notebook [8]. Proofs and other references of all 
the identities can be found in [1]. However, in the unorganized pages of his notebooks [8], Ramanujan recorded many other beautiful identities. Proofs of these identities can be found in [2, 3]. In Section 2, we prove some of these identities by using some other identities of theta-functions. Berndt [2,3] proved these identities via parameterization.

At scattered places in his notebooks [8], Ramanujan recorded several values of his theta-function $\phi(q)$. Proofs of all the values claimed by Ramanujan can be found in [3, Chapter 35]. Berndt and Chan [4] also verified all of Ramanujan's nonelementary values of $\phi\left(e^{-n \pi}\right)$ and found three new values for $n=13,27$, and 63. Kang [6] also calculated some quotients of theta-functions $\phi$ and $\psi$. In Section 3, we give some theorems for the explicit evaluation of the quotients of theta-functions $\phi, \psi$, and $f$, by combining Weber-Ramanujan class invariants with the identities proved in Section 2 and some other identities of theta-functions. Some of these evaluations can be used to find explicit values of the famous Rogers-Ramanujan continued fraction $R(q)$ defined by

$$
R(q):=\frac{q^{1 / 5}}{1}+\frac{q}{1}+\frac{q^{2}}{1}+\frac{q^{3}}{1}+\cdots
$$

where $|q|<1$.

We end this introduction by defining Weber-Ramanujan class invariants $G_{n}$ and $g_{n}$. For $q=\exp (-\pi \sqrt{n})$, where $n$ is a positive rational number, the Weber-Ramanujan class invariants $G_{n}$ and $g_{n}$ are defined by

$$
\begin{aligned}
G_{n} & :=2^{-1 / 4} q^{-1 / 24} \chi(q), \\
g_{n} & :=2^{-1 / 4} q^{-1 / 24} \chi(-q) .
\end{aligned}
$$

2. Theta-function identities. The following identity was recorded by Ramanujan on page 295 of his first notebook [8]. Berndt [3, page 366] proved this by using parameterization. Here we give an alternative proof.

THEOREM 2.1. If $\phi(q), \psi(q)$, and $\chi(q)$ are defined by (1.2), (1.3), and (1.5), respectively, then

$$
\psi^{2}(-q)+5 q \psi^{2}\left(-q^{5}\right)=\frac{\phi^{2}(q)}{\chi(q) \chi\left(q^{5}\right)} .
$$

Proof. From [1, Entry 9(vii), page 258, and Entry 10(v), page 262], we find that

$$
\psi^{2}(q)-q \psi^{2}\left(q^{5}\right)=\frac{\phi\left(-q^{5}\right) f\left(-q^{5}\right)}{x(-q)}
$$

From [1, Entry 24(iii), page 39], we note that

$$
f(q)=\frac{\phi(q)}{x(q)}
$$


From (2.2) and (2.3), we deduce that

$$
\psi^{2}(q)-q \psi^{2}\left(q^{5}\right)=\frac{\phi^{2}\left(-q^{5}\right)}{\chi(-q) \chi\left(-q^{5}\right)} .
$$

Now, we recall from [1, Entry 9(iii), page 258] that

$$
\phi^{2}(q)-\phi^{2}\left(q^{5}\right)=4 q \chi(q) f\left(-q^{5}\right) f\left(-q^{20}\right) .
$$

Replacing $q$ by $-q$ in (2.5), we deduce that

$$
\phi^{2}\left(-q^{5}\right)=\phi^{2}(-q)+4 q \chi(-q) f\left(q^{5}\right) f\left(-q^{20}\right) .
$$

Employing (2.6) in (2.4), we find that

$$
\psi^{2}(q)-q \psi^{2}\left(q^{5}\right)=\frac{\phi^{2}(-q)}{\chi(-q) \chi\left(-q^{5}\right)}+4 q \frac{f\left(q^{5}\right) f\left(-q^{20}\right)}{\chi\left(-q^{5}\right)} .
$$

Again, by [1, Entry 24(iii), page 39], we find that

$$
f\left(-q^{4}\right)=\psi\left(q^{2}\right) \chi\left(-q^{2}\right) .
$$

Using (2.8) in (2.7), we obtain

$$
\psi^{2}(q)-q \psi^{2}\left(q^{5}\right)=\frac{\phi^{2}(-q)}{\chi(-q) \chi\left(-q^{5}\right)}+4 q \frac{f\left(q^{5}\right) \psi\left(q^{10}\right) \chi\left(-q^{10}\right)}{\chi\left(-q^{5}\right)} .
$$

Now, by [1, Entry 24(iv), page 39], we note that

$$
\chi(q) \chi(-q)=\chi\left(-q^{2}\right) .
$$

Thus, from (2.9), we obtain

$$
\psi^{2}(q)-q \psi^{2}\left(q^{5}\right)=\frac{\phi^{2}(-q)}{\chi(-q) \chi\left(-q^{5}\right)}+4 q f\left(q^{5}\right) \psi\left(q^{10}\right) \chi\left(q^{5}\right) .
$$

From [1, Entry 25(iv), page 40], we note that

$$
\phi(q) \psi\left(q^{2}\right)=\psi^{2}(q) .
$$

Employing (2.3) and (2.12), with $q$ replaced by $q^{5}$, we conclude from (2.11) that

$$
\psi^{2}(q)-q \psi^{2}\left(q^{5}\right)=\frac{\phi^{2}(-q)}{\chi(-q) \chi\left(-q^{5}\right)}+4 q \psi^{2}\left(q^{5}\right)
$$

Replacing $q$ by $-q$ in (2.13), we complete the theorem.

The next theorem was recorded by Ramanujan on page 4 of his second notebook [8]. Berndt [2, page 202] proved this theorem by parameterization. Here we give an alternative proof by using some identities of theta-functions. 
THEOREM 2.2. With $\psi(q)$ and $\chi(q)$ defined in (1.3) and (1.5), respectively,

$$
\begin{aligned}
& \frac{\chi^{3}(q)}{\chi\left(q^{3}\right)}=1+3 q \frac{\psi\left(-q^{9}\right)}{\psi(-q)}, \\
& \frac{\chi^{5}(q)}{\chi\left(q^{5}\right)}=1+5 q \frac{\psi^{2}\left(-q^{5}\right)}{\psi^{2}(-q)} .
\end{aligned}
$$

Proof of (2.14). From [1, Chapter 16, Corollary (ii) of Entry 31, page 49], we find that

$$
\psi(q)-q \psi\left(q^{9}\right)=f\left(q^{3}, q^{6}\right)
$$

Using the Jacobi triple product identity, Berndt [1, page 350] proved that

$$
f\left(q, q^{2}\right)=\frac{\phi\left(-q^{3}\right)}{\chi(-q)} .
$$

Replacing $q$ by $q^{3}$ in (2.17) and then using the resultant identity in (2.16), we find that

$$
\psi(q)-q \psi\left(q^{9}\right)=\frac{\phi\left(-q^{9}\right)}{\chi\left(-q^{3}\right)} .
$$

Now, from [1, Corollary (i) of Entry 31, page 49 and Example (v), page 51], we find that

$$
\phi\left(-q^{9}\right)=\phi(-q)+2 q \psi\left(q^{9}\right) \times\left(-q^{3}\right)
$$

Invoking (2.19) in (2.18), we deduce that

$$
\psi(q)-3 q \psi\left(q^{9}\right)=\frac{\phi(-q)}{x\left(-q^{3}\right)} .
$$

Thus,

$$
1-3 q \frac{\psi\left(q^{9}\right)}{\psi(q)}=\frac{\phi(-q)}{\chi\left(-q^{3}\right) \psi(q)}
$$

Now, from [1, Entry 24(iii), page 39], we note that

$$
\chi(q)=\sqrt[3]{\frac{\phi(q)}{\psi(-q)}} .
$$

Replacing $q$ by $-q$ in (2.21) and then using (2.22), we complete the proof of (2.14).

Proof of (2.15). From Theorem 2.1, we find that

$$
1+5 q \frac{\psi^{2}\left(-q^{5}\right)}{\psi^{2}(-q)}=\frac{\phi^{2}(q)}{\chi(q) \chi\left(q^{5}\right) \psi^{2}(-q)} .
$$

Employing (2.22) in (2.23), we arrive at (2.15), which completes the proof. 


\section{Explicit evaluations of theta-functions}

THEOREM 3.1. If $\psi(q), G_{n}$, and $g_{n}$ are defined by (1.3), (1.7), and (1.8), respectively, then

$$
\begin{aligned}
e^{-\pi \sqrt{n}} \frac{\psi\left(-e^{-9 \pi \sqrt{n}}\right)}{\psi\left(-e^{-\pi \sqrt{n}}\right)} & =\frac{1}{3}\left(\sqrt{2} \frac{G_{n}^{3}}{G_{9 n}}-1\right), \\
e^{-\pi \sqrt{n}} \frac{\psi\left(e^{-9 \pi \sqrt{n}}\right)}{\psi\left(e^{-\pi \sqrt{n}}\right)} & =\frac{1}{3}\left(1-\sqrt{2} \frac{g_{n}^{3}}{g_{9 n}}\right) .
\end{aligned}
$$

Proof. From (2.14) and the definition of $G_{n}$ from (1.7), we easily arrive at (3.1). To prove (3.2), we replace $q$ by $-q$ in (2.14) and then use the definition of $g_{n}$ from (1.8).

Since $G_{9 n}$ and $g_{9 n}$ can be calculated from the respective values of $G_{n}$ and $g_{n}$ [5], from the theorem above, we see that the quotients of theta-functions on the left-hand sides can be evaluated if the corresponding values of $G_{n}$ and $g_{n}$ are known. We give a few examples below.

COROLlary 3.2.

$$
e^{-\pi} \frac{\psi\left(-e^{-9 \pi}\right)}{\psi\left(-e^{-\pi}\right)}=\frac{\sqrt[3]{2(\sqrt{3}-1)}-1}{3} .
$$

Proof. Putting $n=1$ in (3.1), we find that

$$
e^{-\pi} \frac{\psi\left(-e^{-9 \pi}\right)}{\psi\left(-e^{-\pi}\right)}=\frac{1}{3}\left(\sqrt{2} \frac{G_{1}^{3}}{G_{9}}-1\right) .
$$

From [3, page 189],

$$
G_{1}=1, \quad G_{9}=\left(\frac{1+\sqrt{3}}{\sqrt{2}}\right)^{1 / 3} .
$$

Employing (3.5) in (3.4) and then simplifying, we complete the proof.

From [1, Entry 11(ii), page 123], we find that

$$
\psi\left(-e^{-\pi}\right)=\phi\left(e^{-\pi}\right) 2^{-3 / 4} e^{\pi / 8} .
$$

Since

$$
\phi\left(e^{-\pi}\right)=\frac{\pi^{1 / 4}}{\Gamma(3 / 4)}
$$

is classical [9], (3.3) and (3.6) provide an explicit evaluation for $\psi\left(-e^{-9 \pi}\right)$.

COROLLARY 3.3.

$$
e^{-\pi \sqrt{5} / 3} \frac{\psi\left(-e^{-3 \pi \sqrt{5}}\right)}{\psi\left(-e^{-\pi \sqrt{5} / 3}\right)}=\frac{(3+\sqrt{5})(\sqrt{5}-\sqrt{3})-2}{6} .
$$


Proof. Putting $n=5 / 9$ in (3.1), we obtain

$$
e^{-\pi \sqrt{5} / 3} \frac{\psi\left(-e^{-3 \pi \sqrt{5}}\right)}{\psi\left(-e^{-\pi \sqrt{5} / 3}\right)}=\frac{1}{3}\left(\sqrt{2} \frac{G_{5 / 9}^{3}}{G_{5}}-1\right) .
$$

Now, from [3, pages 189 and 345], we note that

$$
G_{5}=\left(\frac{1+\sqrt{5}}{2}\right)^{1 / 4}, \quad G_{5 / 9}=(\sqrt{5}+2)^{1 / 4}\left(\frac{\sqrt{5}-\sqrt{3}}{\sqrt{2}}\right)^{1 / 3} .
$$

Employing (3.10) in (3.9) and then simplifying, we arrive at (3.8).

\section{COROLlary 3.4.}

$$
e^{-\pi \sqrt{2}} \frac{\psi\left(e^{-9 \pi \sqrt{2}}\right)}{\psi\left(e^{-\pi \sqrt{2}}\right)}=\frac{1-\sqrt{2} \sqrt[3]{\sqrt{3}-\sqrt{2}}}{3}
$$

Proof. Putting $n=2$ in (3.2), we find that

$$
e^{-\pi \sqrt{2}} \frac{\psi\left(e^{-9 \pi \sqrt{2}}\right)}{\psi\left(e^{-\pi \sqrt{2}}\right)}=\frac{1}{3}\left(1-\sqrt{2} \frac{g_{2}^{3}}{g_{18}}\right) .
$$

From [3, page 200], we note that

$$
g_{2}=1, \quad g_{18}=(\sqrt{2}+\sqrt{2})^{1 / 3} .
$$

Using (3.13) in (3.12), we easily arrive at (3.11).

THeOREM 3.5. With $\psi(q), G_{n}$, and $g_{n}$ defined in (1.3), (1.7), and (1.8), respectively,

$$
\begin{aligned}
e^{-\pi \sqrt{n}} \frac{\psi^{2}\left(-e^{-5 \pi \sqrt{n}}\right)}{\psi^{2}\left(-e^{-\pi \sqrt{n}}\right)} & =\frac{1}{5}\left(2 \frac{G_{n}^{5}}{G_{25 n}}-1\right), \\
e^{-\pi \sqrt{n}} \frac{\psi^{2}\left(e^{-5 \pi \sqrt{n}}\right)}{\psi^{2}\left(e^{-\pi \sqrt{n}}\right)} & =\frac{1}{5}\left(1-2 \frac{g_{n}^{5}}{g_{25 n}}\right) .
\end{aligned}
$$

Proof. From (2.15) and the definition of $G_{n}$ from (1.7), we easily arrive at (3.14). Replacing $q$ by $-q$ in (2.15) and then using the definition of $g_{n}$ from (1.8), we arrive at (3.15).

If the class invariants are known, then we can explicitly find the values of the quotients of the left-hand-side expressions of the theorem. Next we give some examples.

COROllary 3.6 [6].

$$
e^{-\pi} \frac{\psi^{2}\left(-e^{-5 \pi}\right)}{\psi^{2}\left(-e^{-\pi}\right)}=\frac{1}{5 \sqrt{5}+10}
$$

Proof. Putting $n=1$ in (3.14), we find that

$$
e^{-\pi} \frac{\psi^{2}\left(-e^{-5 \pi}\right)}{\psi^{2}\left(-e^{-\pi}\right)}=\frac{1}{5}\left(2 \frac{G_{1}^{5}}{G_{25}}-1\right) .
$$


From [3, page 189],

$$
G_{1}=1, \quad G_{25}=\frac{1+\sqrt{5}}{2} .
$$

Employing (3.18) in (3.17) and then simplifying, we complete the proof.

\section{COROLlary 3.7.}

$$
e^{-\pi / \sqrt{5}} \frac{\psi^{2}\left(-e^{-\sqrt{5} \pi}\right)}{\psi^{2}\left(-e^{-\pi / \sqrt{5}}\right)}=\frac{1}{\sqrt{5}} .
$$

Proof. We put $n=1 / 5$ in (3.14) to obtain

$$
e^{-\pi / \sqrt{5}} \frac{\psi^{2}\left(-e^{-\sqrt{5} \pi}\right)}{\psi^{2}\left(-e^{-\pi / \sqrt{5}}\right)}=\frac{1}{5}\left(2 G_{5}^{4}-1\right) .
$$

Since, from [3, page 189],

$$
G_{5}=\left(\frac{1+\sqrt{5}}{2}\right)^{1 / 4}
$$

we can easily complete the proof by (3.20).

\section{COROLlaRY 3.8 .}

$$
e^{-\pi \sqrt{3 / 5}} \frac{\psi^{2}\left(-e^{-\pi \sqrt{15}}\right)}{\psi^{2}\left(-e^{-\pi \sqrt{3 / 5}}\right)}=\frac{3-\sqrt{5}}{5+\sqrt{5}} .
$$

Proof. Putting $n=3 / 5$ in (3.14), we obtain

$$
e^{-\pi \sqrt{3 / 5}} \frac{\psi^{2}\left(-e^{-\pi \sqrt{15}}\right)}{\psi^{2}\left(-e^{-\pi \sqrt{3 / 5}}\right)}=\frac{1}{5}\left(2 \frac{G_{3 / 5}^{5}}{G_{15}}-1\right) .
$$

Now, from [3, page 341], we note that

$$
G_{15}=2^{-1 / 12}(1+\sqrt{5})^{1 / 3}, \quad G_{3 / 5}=2^{-1 / 12}(\sqrt{5}-1)^{1 / 3} .
$$

Employing (3.24) in (3.23) and then simplifying, we arrive at (3.22).

\section{COROLlary 3.9.}

$$
e^{-\pi \sqrt{2}} \frac{\psi^{2}\left(e^{-5 \pi \sqrt{2}}\right)}{\psi^{2}\left(e^{-\pi \sqrt{2}}\right)}=\frac{1}{5}\left(1-\frac{2}{a}\right),
$$

where

$$
a=g_{50}=\frac{1}{3}\left(1+\left(\frac{5+\sqrt{5}}{4}\right)^{1 / 3}(\sqrt[3]{1+7 \sqrt{5}+6 \sqrt{6}}+\sqrt[3]{1+7 \sqrt{5}-6 \sqrt{6}})\right)
$$


Proof. We put $n=2$ in (3.15) to obtain

$$
e^{-\pi \sqrt{2}} \frac{\psi^{2}\left(e^{-5 \pi \sqrt{2}}\right)}{\psi^{2}\left(e^{-\pi \sqrt{2}}\right)}=\frac{1}{5}\left(1-2 \frac{g_{2}^{5}}{g_{50}}\right)
$$

From [3, page 201],

$$
g_{50}=\frac{1}{3}\left(1+\left(\frac{5+\sqrt{5}}{4}\right)^{1 / 3}(\sqrt[3]{1+7 \sqrt{5}+6 \sqrt{6}}+\sqrt[3]{1+7 \sqrt{5}-6 \sqrt{6}})\right)
$$

Employing (3.13) and (3.28) in (3.27), we complete the proof.

Since for $q=e^{-\pi \sqrt{n}}, n$ positive rational, the explicit formulas for $\phi^{2}\left(q^{5}\right) / \phi^{2}(q)$, $\phi\left(q^{9}\right) / \phi(q)$, and $\phi^{4}\left(q^{3}\right) / \phi^{4}(q)$ are known [3, page 339, (8.11); page 334, (5.7); page 330, (4.5), respectively], namely,

$$
\begin{aligned}
\frac{\phi^{2}\left(e^{-5 \pi \sqrt{n}}\right)}{\phi^{2}\left(e^{-\pi \sqrt{n}}\right)} & =\frac{1}{5}\left(1+2 \frac{G_{25 n}}{G_{n}^{5}}\right), \\
\frac{\phi\left(e^{-9 \pi \sqrt{n}}\right)}{\phi\left(e^{-\pi \sqrt{n}}\right)} & =\frac{1}{3}\left(1+\sqrt{2} \frac{G_{9 n}}{G_{n}^{3}}\right), \\
\frac{\phi^{4}\left(e^{-3 \pi \sqrt{n}}\right)}{\phi^{4}\left(e^{-\pi \sqrt{n}}\right)} & =\frac{1}{9}\left(1+2 \sqrt{2} \frac{G_{9 n}^{3}}{G_{n}^{9}}\right),
\end{aligned}
$$

we now derive some identities by which the corresponding values of the quotients $\psi^{2}\left(-q^{5}\right) / \psi^{2}(-q), \psi\left(-q^{9}\right) / \psi(-q)$, and $\psi^{4}\left(-q^{3}\right) / \psi^{4}(-q)$ can be found.

THEOREM 3.10 [7]. If $\phi(q)$ and $\psi(q)$ are defined by (1.2) and (1.3), respectively, then

$$
q \frac{\psi^{2}\left(-q^{5}\right)}{\psi^{2}(-q)}=\frac{1-\phi^{2}\left(q^{5}\right) / \phi^{2}(q)}{\left(5 \phi^{2}\left(q^{5}\right) / \phi^{2}(q)\right)-1} .
$$

Proof. We replace $q$ by $-q$ in (2.4) and then divide the resulting identity by (2.1) to obtain

$$
\frac{\phi^{2}\left(q^{5}\right)}{\phi^{2}(q)}=\frac{\psi^{2}(-q)+q \psi^{2}\left(-q^{5}\right)}{\psi^{2}(-q)+5 q \psi^{2}\left(-q^{5}\right)} .
$$

This is indeed equivalent to (3.32).

THEOREM 3.11. With $\phi(q)$ and $\psi(q)$ defined in (1.2) and (1.3), respectively,

$$
q \frac{\psi\left(-q^{9}\right)}{\psi(-q)}=\frac{1-\phi\left(q^{9}\right) / \phi(q)}{\left(3 \phi\left(q^{9}\right) / \phi(q)\right)-1} .
$$

Proof. Replace $q$ by $-q$ in (2.18) and (2.20) and then, dividing the first resulting identity by the second, we find that

$$
\frac{\phi(q)}{\phi\left(q^{9}\right)}=\frac{\psi(-q)+q \psi\left(-q^{9}\right)}{\psi(-q)+3 q \psi\left(-q^{9}\right)} .
$$

It is now easy to see that (3.35) and (3.34) are equivalent. 
THEOREM 3.12. With $\phi(q)$ and $\psi(q)$ defined in (1.2) and (1.3), respectively,

$$
1+9 q \frac{\psi^{4}\left(-q^{3}\right)}{\psi^{4}(-q)}=\frac{8}{\left(9 \phi^{4}\left(q^{3}\right) / \phi^{4}(q)\right)-1} .
$$

Proof. From Theorem 3.11, we note that

$$
1+3 q \frac{\psi\left(-q^{9}\right)}{\psi(-q)}=\frac{2}{\left(3 \phi\left(q^{9}\right) / \phi(q)\right)-1} .
$$

From the third equality of [1, Entry 1(ii), page 345] and the second equality of [1, Entry 1(iii), page 345], we note that

$$
\begin{aligned}
1+3 q \frac{\psi\left(-q^{9}\right)}{\psi(-q)} & =\left(1+9 q \frac{\psi^{4}\left(-q^{3}\right)}{\psi^{4}(-q)}\right)^{1 / 3}, \\
3 \frac{\phi\left(q^{9}\right)}{\phi(q)}-1 & =\left(9 \frac{\phi^{4}\left(q^{3}\right)}{\phi^{4}(q)}-1\right)^{1 / 3},
\end{aligned}
$$

respectively. Employing (3.38) in (3.37) and then cubing the resultant identity, we complete the proof.

\section{COROLLARY 3.13.}

$$
e^{-\pi} \frac{\psi^{4}\left(-e^{-3 \pi}\right)}{\psi^{4}\left(-e^{-\pi}\right)}=\frac{2-\sqrt{3}}{3 \sqrt{3}}
$$

Proof. It is known from [3, page 327] (or can be found easily from (3.31)) that

$$
\frac{\phi^{4}\left(e^{-3 \pi}\right)}{\phi^{4}\left(e^{-\pi}\right)}=\frac{1}{6 \sqrt{3}-9}
$$

The proof of the corollary now follows immediately by putting $q=e^{-\pi}$ in Theorem 3.12 and then using (3.40).

Now, from [1, Entries 24(ii) and 24(iv), page 39], we note that

$$
\begin{aligned}
f^{3}(q) & =\phi^{2}(q) \psi(-q), \\
f^{3}\left(-q^{2}\right) & =\phi(q) \psi^{2}(-q) .
\end{aligned}
$$

From (3.41), we find the following quotients of $f$ in terms of $\phi$ and $\psi$ :

$$
\begin{aligned}
& F_{1}(q):=\frac{f^{6}(q)}{q f^{6}\left(q^{5}\right)}=\frac{\psi^{2}(-q)}{q \psi^{2}\left(-q^{5}\right)} \times \frac{\phi^{4}(q)}{\phi^{4}\left(q^{5}\right)}, \\
& F_{2}(q):=\frac{f^{6}\left(-q^{2}\right)}{q^{2} f^{6}\left(-q^{10}\right)}=\frac{\phi^{2}(q)}{\phi^{2}\left(q^{5}\right)} \times \frac{\psi^{4}(-q)}{q^{2} \psi^{4}\left(-q^{5}\right)} .
\end{aligned}
$$


The values of $F_{1}(q)$ and $F_{2}(q)$ can be determined explicitly for $q=e^{-\pi \sqrt{n}}$ by employing Theorem 3.5 and (3.29). We give a couple of examples below.

COROLLARY 3.14.

$$
\begin{aligned}
& F_{1}\left(e^{-\pi / \sqrt{5}}\right)=5 \sqrt{5}, \\
& F_{2}\left(e^{-\pi / \sqrt{5}}\right)=5 \sqrt{5} .
\end{aligned}
$$

Proof. As in Corollary 3.7, by putting $n=1 / 5$ in (3.29), it can be easily seen that

$$
\frac{\phi^{2}\left(e^{-\sqrt{5} \pi}\right)}{\phi^{2}\left(e^{-\pi / \sqrt{5}}\right)}=\frac{1}{\sqrt{5}} .
$$

Putting $q=e^{-\pi / \sqrt{5}}$ in (3.42) and then employing (3.44) and Corollary 3.7, we complete the proof.

\section{COROLLARY 3.15 .}

$$
\begin{aligned}
& F_{1}\left(e^{-\pi \sqrt{3 / 5}}\right)=\frac{5(5+\sqrt{5})}{2}, \\
& F_{2}\left(e^{-\pi \sqrt{3 / 5}}\right)=\frac{5(25+11 \sqrt{5})}{2} .
\end{aligned}
$$

Proof. As in Corollary 3.8, by putting $n=3 / 5$ in (3.29), it can be easily seen that

$$
\frac{\phi^{2}\left(e^{-\sqrt{15} \pi}\right)}{\phi^{2}\left(e^{-\pi \sqrt{3 / 5}}\right)}=\frac{2}{5-\sqrt{5}} .
$$

Putting $q=e^{-\pi \sqrt{3 / 5}}$ in (3.42) and then employing (3.46) and Corollary 3.8, we complete the proof.

Now, for the explicit evaluation of $R(q)$ defined in (1.6), we note from [6] that

$$
\begin{aligned}
\frac{1}{R^{5}\left(q^{2}\right)}-11-R^{5}\left(q^{2}\right) & =\frac{f^{6}\left(-q^{2}\right)}{q^{2} f^{6}\left(-q^{10}\right)}, \\
\frac{1}{S^{5}(q)}+11-S^{5}(q) & =\frac{f^{6}(q)}{q f^{6}\left(q^{5}\right)},
\end{aligned}
$$

where $S(q)=-R(-q)$.

From (3.47) and (3.42), we see that to find the explicit values of $R\left(q^{2}\right)$ and $S(q)$, for $q=e^{-\pi \sqrt{n}}$, it is enough to find $F_{1}(q)$ and $F_{2}(q)$. See [6].

ACKNOWLEDGMENT. The authors thank Bruce C. Berndt for sending some of his books and reprints. They also thank the referee for helpful suggestions.

\section{REFERENCES}

[1] B. C. Berndt, Ramanujan's Notebooks. Part III, Springer-Verlag, New York, 1991.

[2] _ Ramanujan's Notebooks. Part IV, Springer-Verlag, New York, 1994.

[3] _ Ramanujan's Notebooks. Part V, Springer-Verlag, New York, 1998. 
[4] B. C. Berndt and H. H. Chan, Ramanujan's explicit values for the classical theta-function, Mathematika 42 (1995), no. 2, 278-294.

[5] B. C. Berndt, H. H. Chan, and L.-C. Zhang, Ramanujan's class invariants and cubic continued fraction, Acta Arith. 73 (1995), no. 1, 67-85.

[6] S.-Y. Kang, Ramanujan's formulas for the explicit evaluation of the Rogers-Ramanujan continued fraction and theta-functions, Acta Arith. 90 (1999), no. 1, 49-68.

[7] Some theorems on the Rogers-Ramanujan continued fraction and associated theta function identities in Ramanujan's lost notebook, Ramanujan J. 3 (1999), no. 1, 91111.

[8] S. Ramanujan, Notebooks. Vols. 1, 2, Tata Institute of Fundamental Research, Bombay, 1957.

[9] E. T. Whittaker and G. N. Watson, A Course of Modern Analysis, Cambridge Mathematical Library, Cambridge University Press, Cambridge, 1996.

Nayandeep Deka Baruah: Department of Mathematical Sciences, Tezpur University, Napaam 784 028, Sonitpur, Assam, India

E-mail address: nayan@tezu.ernet.in

P. Bhattacharyya: Department of Mathematical Sciences, Tezpur University, Napaam 784028 , Sonitpur, Assam, India

E-mail address: pb@tezu.ernet.in 


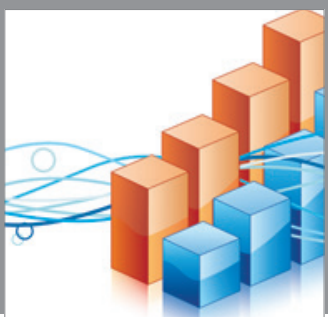

Advances in

Operations Research

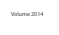

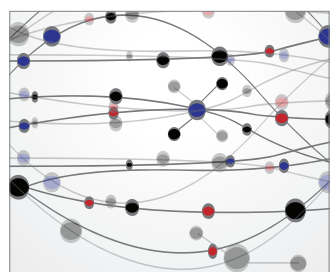

\section{The Scientific} World Journal
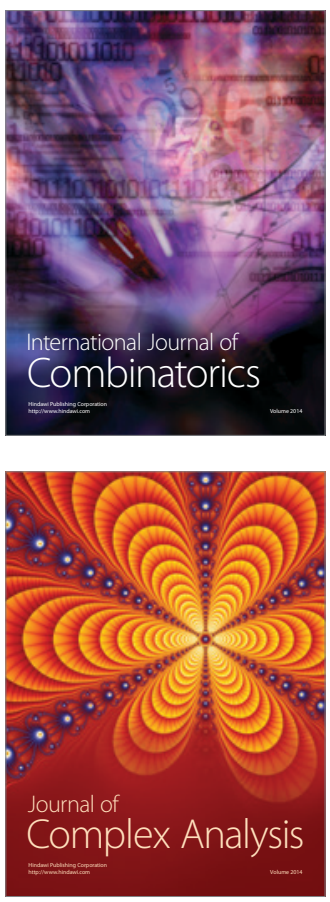

International Journal of

Mathematics and

Mathematical

Sciences
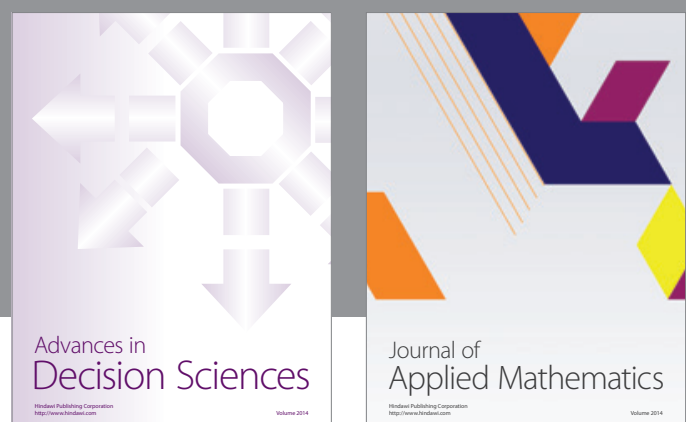

Journal of

Applied Mathematics
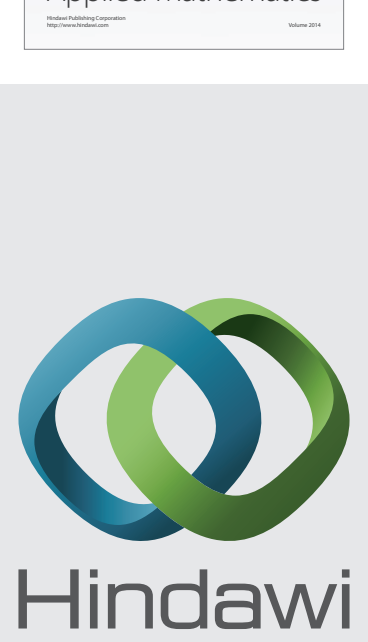

Submit your manuscripts at http://www.hindawi.com
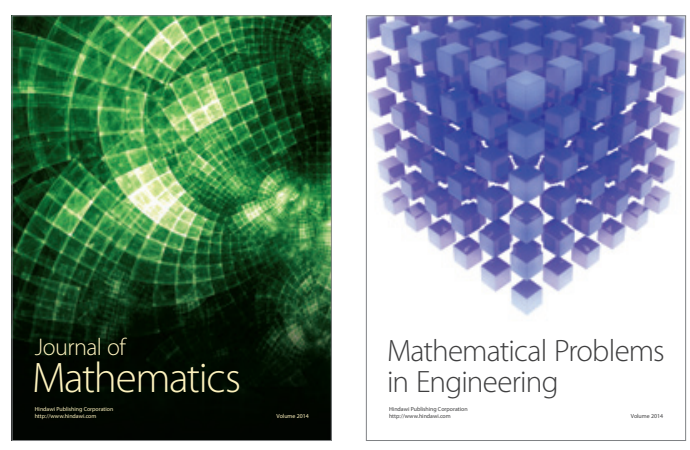

Mathematical Problems in Engineering
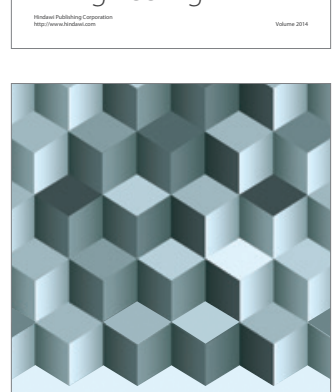

Journal of

Function Spaces
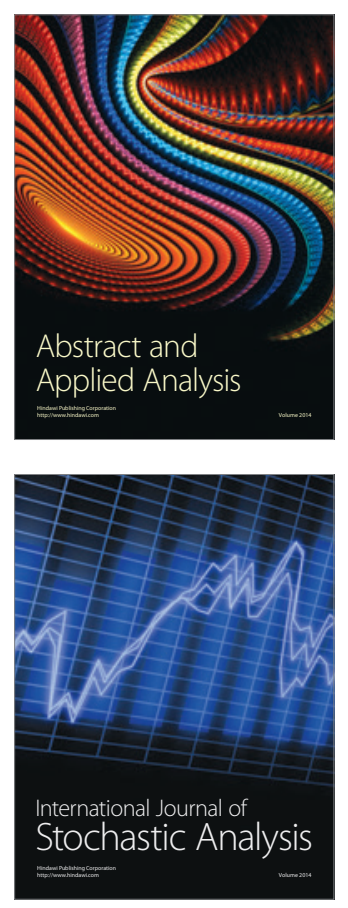

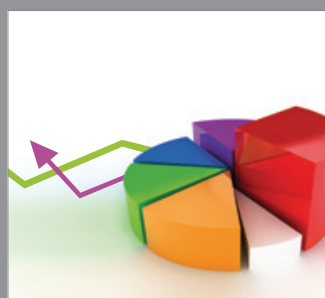

ournal of

Probability and Statistics

Promensencen
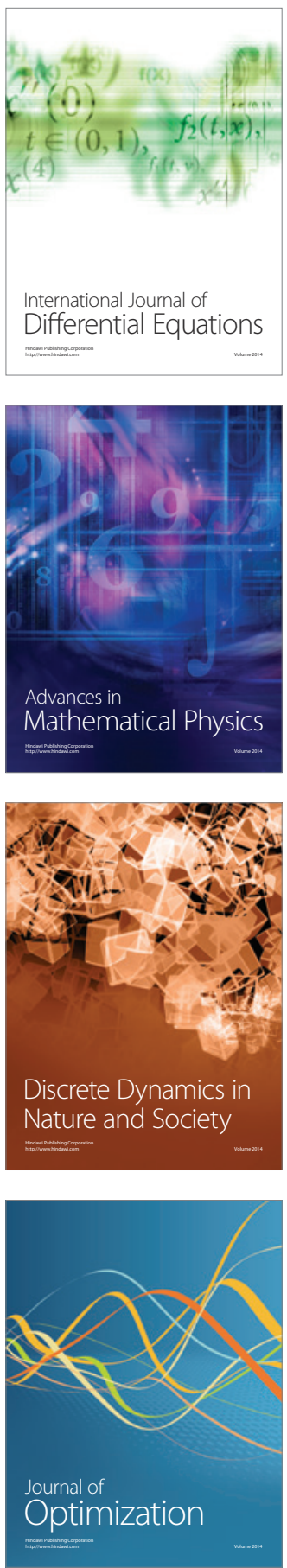